\title{
Hidden $U(1)$ gauge symmetry realizing a neutrinophilic two-Higgs-doublet model with dark matter
}

\author{
Takaaki Nomura ${ }^{1, *}$ and Hiroshi Okada ${ }^{2, \dagger}$ \\ ${ }^{1}$ School of Physics, KIAS, Seoul 02455, Korea \\ ${ }^{2}$ Physics Division, National Center for Theoretical Sciences, Hsinchu, Taiwan 300
}

(Received 15 February 2018; published 30 April 2018)

\begin{abstract}
We propose a neutrinophilic two-Higgs-doublet model with hidden local $U(1)$ symmetry, where active neutrinos are Dirac type, and a fermionic dark matter (DM) candidate is naturally induced as a result of remnant symmetry even after the spontaneous symmetry breaking. In addition, a physical Goldstone boson arises as a consequence of two types of gauge singlet bosons and contributes to the DM phenomenologies as well as an additional neutral gauge boson. Then, we analyze the relic density of DM within the safe range of direct detection searches and show the allowed region of dark matter mass.
\end{abstract}

DOI: 10.1103/PhysRevD.97.075038

\section{INTRODUCTION}

The neutrinophilic two-Higgs-doublet model (NTHDM) [1-4] is one of the appropriate explanations to relax the neutrino Yukawa coupling where one of Higgs doublets has only the neutrino Yukawa interaction and develops a tiny vacuum expectation value (VEV) to generate the neutrino masses. To discriminate the neutrinophilic Higgs doublet from the standard model (SM)-like Higgs doublet, one usually imposes an additional symmetry such as a global and/or gauged one [5-12], and this symmetry often plays a role in assuring stability of a dark matter (DM) candidate.

We can construct a NTHDM with extra $U(1)$ gauge symmetry assigning its charge to right-handed neutrinos and one Higgs doublet so that this Higgs doublet only has Yukawa couplings associated with right-handed neutrino and lepton doublets. In such a case, other SM fermions would be required to have extra $U(1)$ charges for anomaly cancellation as in the $U(1)_{B-L}$ model. Alternatively, we find that we can cancel the gauge anomaly among only SM singlet fermions by adding extra fermions in addition to right-handed neutrinos and extra $U(1)$ gauge symmetry is a hidden gauge symmetry. As a result of the gauge symmetry, the lightest extra fermions are stable and can be good DM candidates.

In this paper, we introduce a local hidden $U(1)$ symmetry $\left[U(1)_{H}\right]$, and neutrino masses are Dirac type [9]

\footnotetext{
*nomura@kias.re.kr

†macokada3hiroshi@cts.nthu.edu.tw
}

Published by the American Physical Society under the terms of the Creative Commons Attribution 4.0 International license. Further distribution of this work must maintain attribution to the author(s) and the published article's title, journal citation, and DOI. Funded by SCOAP . induced by the VEV of the neutrinophilic Higgs doublet, which has $U(1)_{H}$ charge. After spontaneous symmetry breaking, a fermionic DM candidate arises as a result of remnant symmetry. Simultaneously, a physical Goldstone boson (GB) can contribute to the DM phenomenologies as well as an additional neutral gauge boson, as a result of introducing two types of gauge singlet bosons that break $U(1)_{H}$. We then show the observed relic density of DM can be explained either by GB interactions or $Z^{\prime}$ interactions.

This paper is organized as follows. In Sec. II, we show our model and formulate the boson sector, fermion sector, and dark matter sector. Then, we analyze DM through the relic density and discuss the allowed region in terms of DM mass. Finally, we conclude and discuss in Sec. III.

\section{MODEL SETUP AND PHENOMENOLOGIES}

First of all, we introduce a $U(1)_{H}$ hidden gauge symmetry and add six right-handed neutral fermions $\left(N_{R_{i}}, N_{R_{3}}\right)$ and $\nu_{R_{a}}$ with $i=1,2$ and a $=1-3$, which are charged under the new gauge symmetry. As we discuss below, gauge anomalies are canceled among these additional fermions, and active neutrinos are Dirac type with right-handed neutrinos $\nu_{R_{a}}$. In the scalar sector, we introduce an isospin doublet scalar $\Phi$ that has $U(1)_{H}$ charge 1 and two isospin singlet bosons $\left(\varphi, \varphi^{\prime}\right)$ with $U(1)_{H}$ charges $(1,8)$. Here, $H$ is expected to be the SM-like Higgs doublet field. All the field contents and their assignments are summarized in Table I. Then, one finds the relevant Lagrangian associated with the lepton Yukawa interactions and scalar potential as

$$
\begin{aligned}
-\mathcal{L}_{\text {Lepton }}= & y_{\ell_{a}} \bar{L}_{L_{a}} e_{R_{a}} H+y_{\nu_{a b}} \bar{L}_{L_{a}} \tilde{\Phi} \nu_{R_{b}}+y_{\varphi_{i}} \varphi^{*} \bar{N}_{R_{i}}^{c} N_{R_{3}} \\
& +y_{\varphi_{i j}^{\prime}} \varphi^{\prime} \bar{N}_{R_{i}}^{c} N_{R_{j}}+\text { c.c. }
\end{aligned}
$$


TABLE I. Field contents of bosons and fermions and their charge assignments under $S U(2)_{L} \times U(1)_{Y} \times U(1)_{H}$ in the lepton sector, where $a=1-3$ and $i=1,2$ are flavor indices.

\begin{tabular}{lccccrrrrr}
\hline \hline Fields & $\Phi$ & $H$ & $\varphi$ & $\varphi^{\prime}$ & $L_{L_{a}}$ & $e_{R_{a}}$ & $N_{R_{i}}$ & $N_{R_{3}}$ & $\nu_{R_{a}}$ \\
\hline$S U(2)_{L}$ & $\mathbf{2}$ & $\mathbf{2}$ & $\mathbf{1}$ & $\mathbf{1}$ & $\mathbf{2}$ & $\mathbf{1}$ & $\mathbf{1}$ & $\mathbf{1}$ & $\mathbf{1}$ \\
$U(1)_{Y}$ & $\frac{1}{2}$ & $\frac{1}{2}$ & 0 & 0 & $-\frac{1}{2}$ & -1 & 0 & 0 & 0 \\
$U(1)_{H}$ & 1 & 0 & 1 & 8 & 0 & 0 & -4 & 5 & 1 \\
\hline \hline
\end{tabular}

$$
\begin{aligned}
V= & -\mu_{H}^{2} H^{\dagger} H-\mu_{\Phi}^{2} \Phi^{\dagger} \Phi-\mu_{\varphi}^{2} \varphi^{\dagger} \varphi-\mu_{\varphi^{\prime}}^{2} \varphi^{\dagger} \varphi^{\prime} \\
& +\lambda_{1}\left(\Phi^{\dagger} \Phi\right)^{2}+\lambda_{2}\left(H^{\dagger} H\right)^{2}+\lambda_{\varphi}\left(\varphi^{\dagger} \varphi\right)^{2}+\lambda_{\varphi^{\prime}}\left(\varphi^{\prime \dagger} \varphi^{\prime}\right)^{2} \\
& +\lambda_{3}\left(H^{\dagger} H\right)\left(\Phi^{\dagger} \Phi\right)+\lambda_{4}\left(H^{\dagger} \Phi\right)\left(\Phi^{\dagger} H\right) \\
& +\lambda_{H \varphi}\left(H^{\dagger} H\right)\left(\varphi^{\dagger} \varphi\right)+\lambda_{H \varphi^{\prime}}\left(H^{\dagger} H\right)\left(\varphi^{\prime \dagger} \varphi^{\prime}\right) \\
& +\lambda_{\Phi \varphi}\left(\Phi^{\dagger} \Phi\right)\left(\varphi^{\dagger} \varphi\right)+\lambda_{\Phi \varphi^{\prime}}\left(\Phi^{\dagger} \Phi\right)\left(\varphi^{\dagger} \varphi\right) \\
& +\lambda_{\varphi \varphi^{\prime}}\left(\varphi^{\dagger} \varphi\right)\left(\varphi^{\prime \dagger} \varphi^{\prime}\right)-\mu_{0}\left[\left(\Phi^{\dagger} H\right) \varphi+\text { c.c. }\right],
\end{aligned}
$$

where $\tilde{\Phi} \equiv\left(i \sigma_{2}\right) \Phi^{*}$ with $\sigma_{2}$ being the second Pauli matrix, $a$ runs over 1 to 3 , and $i$ and $j$ run over 1 to 2 . The first term of the Yukawa coupling provides the SM charged-lepton masses, while the second term induces the active neutrino masses of Dirac type. The term $\mu_{0}$ plays a role in forbidding a massless Goldstone boson appearing from Higgs doublets after spontaneous gauge symmetry breaking [3]. Note that we have $Z_{2}$ symmetry even after scalar fields developing VEVs where extra fermions $\left\{N_{R_{i}}, N_{R_{3}}\right\}$ are $Z_{2}$ odd and the other particles are $Z_{2}$ even at renormalizable level. ${ }^{1}$

Here, we check anomaly cancellations for new gauge symmetry in the model. In our case, we need to check only $U(1)_{H}$ and $\left[U(1)_{H}\right]^{3}$ anomalies since all the $U(1)_{H}$ charged fermions are SM singlet. We then find

$$
\begin{aligned}
U(1)_{H} & : 1+1+1-4-4+5=0 \\
{\left[U(1)_{H}\right]^{3}: } & (1)^{3}+(1)^{3}+(1)^{3}+(-4)^{3}+(-4)^{3}+(5)^{3}=0 .
\end{aligned}
$$

Therefore, our charge assignment is anomaly free.

The scalar fields are parametrized as

$$
\begin{array}{ll}
H=\left[\begin{array}{c}
w^{+} \\
\frac{v_{H}+h+i z}{\sqrt{2}}
\end{array}\right], \quad \Phi=\left[\begin{array}{c}
\phi^{+} \\
\frac{v_{\phi}+\phi_{R}+i \phi_{I}}{\sqrt{2}}
\end{array}\right], \\
\varphi=\frac{v_{\varphi}+\varphi_{R}}{\sqrt{2}} e^{i \frac{\alpha}{v_{\varphi}}}, \quad \varphi^{\prime}=\frac{v_{\varphi^{\prime}}+\varphi_{R}^{\prime}}{\sqrt{2}} e^{i \frac{\alpha^{\prime}}{v_{\varphi^{\prime}}}},
\end{array}
$$

where the lightest mass eigenstate after diagonalizing the matrix in the basis of $\left(w^{ \pm}, \phi^{ \pm}\right)$, which is massless, is

\footnotetext{
${ }^{1}$ At the nonrenormalizable level, there exists a dimension-6 operator of $\bar{\nu}_{R_{a}}^{c} N_{R_{3}}\left(\varphi^{\prime}\right)^{*} \varphi^{2}$. We consider such a term highly suppressed by sufficiently a large cutoff scale as well as its coupling and suppose not to affect the stability of DM and phenomenology.
}

absorbed by the SM singly charged gauge boson $W^{ \pm}$and 2 degrees of freedom in the $C P$-odd boson sector $\left(z, \phi_{I}, \alpha, \alpha^{\prime}\right)$ are also absorbed by the neutral SM gauge boson $Z$ and $U(1)_{H}$ gauge boson $Z^{\prime 2} ; z$ is dominantly a Nambu-Goldstone (NG) boson absorbed by $Z$, and one linear combination of $\left\{\alpha, \alpha^{\prime}\right\}$ is absorbed by $Z^{\prime}$ as discussed below. The nonzero VEVs of scalar fields can be obtained from the condition $\partial V / \partial v_{H, \phi, \varphi, \varphi^{\prime}}=0$. Then, we can simply obtain

$$
\begin{aligned}
v_{\varphi} \simeq \sqrt{\frac{\mu_{\varphi}^{2}}{\lambda_{\varphi}}}, \quad v_{\varphi} & \simeq \sqrt{\frac{\mu_{\varphi^{\prime}}^{2}}{\lambda_{\varphi^{\prime}}}}, \quad v_{H} \simeq \sqrt{\frac{\mu_{H}^{2}}{\lambda_{H}}}, \\
v_{\phi} & \simeq \frac{\sqrt{2} \mu_{0} v_{H} v_{\varphi}}{-2 \mu_{\Phi}^{2}+\left(\lambda_{3}+\lambda_{4}\right) v_{H}^{2}},
\end{aligned}
$$

where we assumed couplings in the potential $\left\{\lambda_{H \varphi}\right.$, $\left.\lambda_{H \varphi^{\prime}}, \lambda_{\Phi \varphi}, \lambda_{\Phi \varphi^{\prime}}, \lambda_{\varphi \varphi^{\prime}}\right\}$ and $v_{\phi}$ to be sufficiently small, and we require $\left\{\mu_{H}^{2}, \mu_{\varphi}^{2}, \mu_{\varphi^{\prime}}^{2}, \mu_{0}\right\}>0$ and $-2 \mu_{\Phi}^{2}+\left(\lambda_{3}+\right.$ $\left.\lambda_{4}\right) v_{H}^{2}>0$ to make all VEVs positive. Note that $v_{\phi}$ is expected to be tiny in order to generate active neutrino mass, which can be realized taking a tiny $\mu_{0}$ value $[3,9]$. One thus finds that $v \equiv \sqrt{v_{H}^{2}+v_{\phi}^{2}} \sim v_{H}$. The mass matrix squared of a singly charged boson is diagonalized by the mixing matrix as

$$
O \equiv\left[\begin{array}{cc}
c_{\beta} & s_{\beta} \\
-s_{\beta} & c_{\beta}
\end{array}\right], \quad s_{\beta}=\frac{v_{\phi}}{\sqrt{v_{\phi}^{2}+v_{H}^{2}}},
$$

where we define $\operatorname{Diag}\left(0, m_{H^{ \pm}}^{2}\right)=O m^{2}\left(w^{ \pm}, \phi^{ \pm}\right) O^{T}$. Therefore, we obtain

$$
\phi^{ \pm} \simeq H^{ \pm}
$$

The mass of the charged Higgs boson is given by

$$
m_{\phi^{ \pm}}^{2} \simeq-\mu_{\Phi}^{2}+\frac{1}{2}\left(\lambda_{3}-\lambda_{4}\right) v_{H}^{2} .
$$

Then, $\mu_{\Phi}^{2}$ is further constrained, requiring $m_{\phi^{ \pm}}^{2}>0$ in addition to the condition for obtaining a positive VEV of $\Phi$. On the other hand, the mass squared matrix of the $C P$-odd boson is in the basis of $\left(z, \phi_{I}, \alpha, \alpha^{\prime}\right)$. After diagonalizing the mass matrix, we obtain one massive $C P$-odd scalar, two NG bosons absorbed by $Z$ and $Z^{\prime}$ bosons, and one massless physical Goldstone boson. We can identify the massive $C P$-odd scalar as $\phi_{I}$, the mass of which is given by

$$
m_{\phi_{I}}^{2} \simeq-\mu_{\Phi}^{2}+\frac{1}{2}\left(\lambda_{3}+\lambda_{4}\right) v_{H}^{2}
$$

\footnotetext{
${ }^{2}$ Since the structure of the scalar sector is more or less the same as the one in Ref. [9], we minimally explain properties of the scalar bosons.
} 
In general, scalar bosons $\left\{\phi_{R}, \phi_{I}, \phi^{ \pm}\right\}$mix with other scalar degrees of freedom that have the same quantum number. However, those mixings are highly suppressed in our scenario in which $v_{\phi}$ is assumed to be tiny in realizing neutrino mass. For example, if we take $y_{\nu}=10^{-6}\left(\sim m_{e} / v\right)$, the required value of $v_{\phi}$ is less than $\sim 100 \mathrm{KeV}$ as $m_{\nu} \sim y_{\nu} v_{\phi}$, and the mixing effect is roughly given by $v_{\phi} / m_{\text {scalar }}$, which is negligibly tiny taking the $m_{\text {scalar }}=\mathcal{O}(100) \mathrm{GeV}$ scale.

The NG boson absorbed by the $Z^{\prime}$ and physical Goldstone bosons is written in terms of a linear combination of $\alpha$ and $\alpha^{\prime}$, where the mixing angle is determined by relative sizes of VEVs of $\varphi$ and $\varphi^{\prime}$. We then obtain NG and physical Goldstone modes denoted by $\alpha_{N G}$ and $\alpha_{G}$ such that ${ }^{3}$

$$
\begin{gathered}
\alpha_{N G}=c_{X} \alpha+s_{X} \alpha^{\prime}, \quad \alpha_{G}=-s_{X} \alpha+c_{X} \alpha^{\prime}, \\
c_{X} \equiv \cos X=\frac{v_{\varphi}}{\sqrt{v_{\varphi}^{2}+64 v_{\varphi^{\prime}}^{2}}}, \\
s_{X} \equiv \sin X=\frac{8 v_{\varphi^{\prime}}}{\sqrt{v_{\varphi}^{2}+64 v_{\varphi^{\prime}}^{2}}} .
\end{gathered}
$$

Notice that the existence of this physical Goldstone boson does not cause serious problem in particle physics or cosmology since it does not couple to SM particles directly and decouples from thermal bass in the early Universe.

The extra gauge boson $Z^{\prime}$ obtains mass after $U(1)_{H}$ symmetry breaking as

$$
m_{Z^{\prime}}^{2}=g_{H}^{2}\left(v_{\varphi}^{2}+64 v_{\varphi^{\prime}}^{2}\right),
$$

where $g_{H}$ denotes the gauge couplings for $U(1)_{H}$ gauge symmetry. Note that we can have $Z-Z^{\prime}$ mixing through the VEV of $\Phi$ since it has both electroweak and $U(1)_{H}$ charge. In our case, however, it is negligibly small due to small $v_{\phi}$, where mixing is suppressed by the $\left(v_{\phi} / m_{Z^{\prime}}\right)^{2}$ factor.

Inserting tadpole conditions, the mass matrix for $C P$ even boson in basis of $\left(h, \phi_{R}, \varphi_{R}, \varphi_{R}^{\prime}\right)$ with nonzero VEVs is defined by $m_{R}^{2}$. Then the mixing matrix $O_{R}$ to diagonalize the mass matrix is defined by $m_{h_{a}}=O_{R} m_{R}^{2} O_{R}^{T}$ and $\left(h, \phi_{R}, \varphi_{R}, \varphi_{R}^{\prime}\right)^{T}=O_{R}^{T} h_{a}$ where $m_{h_{a}}$ is diagonal mass matrix and the mass eigenstate is $h_{a}(a=1-4)$. Here, $h_{1} \equiv h_{\mathrm{SM}}^{T}$ is the SM Higgs; therefore, $m_{h_{1}}=125 \mathrm{GeV}$. In addition, we assume mixing among SM Higgs and other $C P$-even scalars are small to avoid experimental constraints for simplicity.

\section{A. Fermion sector}

First of all, we formulate the mass matrix of the SM leptons. The masses for charged leptons are induced via $y_{\ell}$ after symmetry breaking, and active neutrino masses are also done via the $y_{\nu}$ term where neutrinos are supposed to

\footnotetext{
${ }^{3}$ Derivation of these states is summarized in the Appendix.
}

be Dirac type fermions. Their masses are symbolized by $m_{\ell_{a}} \equiv v_{H} y_{\ell_{a}} / \sqrt{2}$ and $m_{\nu_{a b}} \equiv v_{\phi} y_{\nu_{a b}} / \sqrt{2}$. Since the charged-lepton mass matrix is diagonal, the neutrino mixing matrix $V$ arises from the neutrino mass matrix squared; $\left(m_{\nu}^{2}\right)_{a b}=\sum_{c=1-3}\left(m_{\nu_{a c}} m_{\nu_{c b}}^{\dagger}\right)$, where $V$ is measured by the neutrino oscillation data [13]. Notice here that three active neutrinos can have nonzero mass due to the rank-3 matrix. In our scenario, we take $y_{\nu}$ to not be very large such as $y_{\nu} \sim 10^{-6}\left(\sim m_{e} / v\right)$. Note that our righthanded neutrinos decouple from the thermal bath sufficiently earlier than left-handed neutrinos since $y_{\nu}$ coupling is small and $Z^{\prime}$ mass is heavier than the electroweak scale. Thus, they do not affect cosmological issues such as big bang nucleosynthesis.

Then, we formulate the mass matrix of exotic Majorana fermions $M_{N}$ in the basis of $\left(N_{R_{1}}, N_{R_{2}}, N_{R_{3}}\right)^{T}$, which is given by

$$
M_{N} \equiv\left[\begin{array}{ccc}
m_{11} & m_{12} & M_{1} \\
m_{12} & m_{22} & M_{2} \\
M_{1} & M_{2} & 0
\end{array}\right],
$$

after spontaneous $U(1)$ breaking, where $m_{i j}\left(=m_{j i}\right) \equiv$ $y_{\varphi_{i j}^{\prime}} v_{\varphi^{\prime}} / \sqrt{2}$ and $M_{i} \equiv y_{\varphi_{i}} v_{\varphi} / \sqrt{2}$, with $i, j=1$, 2. Then, $M_{N}$ is diagonalized by $D\left(M_{\psi_{1}}, M_{\psi_{2}}, M_{\psi_{3}}\right)=V_{N} M_{N} V_{N}^{T}$. Thus, one finds $\left(N_{R_{1}}, N_{R_{2}}, N_{R_{3}}\right)^{T} \equiv V_{N}^{T}\left(\psi_{1}, \psi_{2}, \psi_{3}\right)^{T}$, where $V_{N}$ is a unitary mixing matrix in general. Here, we take $N_{R_{1}}$ as the lightest mass eigenstate, and it is a stable particle due to the remnant $Z_{2}$ symmetry as discussed above. Thus, we writhe $X_{R} \equiv \psi_{1}$ and $M_{X} \equiv M_{\psi_{1}}$ for our DM candidate in the following analysis.

\section{B. Dark matter}

In this subsection, we discuss a dark matter candidate, $X_{R}$. First, we assume the contribution from the Higgs mediating interaction is negligibly small and DM annihilation processes are dominated by the gauge interaction with $Z^{\prime}$ and/or GB $\alpha_{G}$; we can thus easily avoid the constraints from direct detection searches such as LUX [14], XENON1T [15], and PandaX-II [16].

We have annihilation modes with Yukawa and kinetic terms to explain the relic density of DM, $\Omega h^{2} \approx 0.12$ [17], and their relevant Lagrangian in the basis of the mass eigenstate is found to be

$$
\begin{aligned}
-\mathcal{L} \supset & \frac{1}{2} Q_{H}^{X} g_{H} \bar{X} \gamma^{\mu} \gamma_{5} X Z_{\mu}^{\prime}+g_{H} Q_{H}^{\nu} \bar{\gamma} \gamma^{\mu} P_{R} \nu Z_{\mu}^{\prime} \\
& +i \frac{\tilde{M}_{1 \beta}}{\tilde{v}_{\varphi \varphi^{\prime}}} \bar{X} P_{R} \psi_{\beta} \alpha_{G}+\text { c.c. } \\
& +i g_{H} Z^{\prime \mu}\left(\partial_{\mu} H^{-} H^{+}-H^{-} \partial_{\mu} H^{+}\right) \\
& +g_{H} Z^{\prime \mu}\left(\partial_{\mu} \phi_{I} \phi_{R}-\phi_{I} \partial_{\mu} \phi_{R}\right),
\end{aligned}
$$


$\frac{\tilde{M}_{\alpha \beta}}{\tilde{v}_{\varphi \varphi^{\prime}}} \equiv \sum_{i=1,2} \frac{M_{i}}{v_{\varphi}} s_{X}\left(V_{N}\right)_{\alpha i}\left(V_{N}^{T}\right)_{3 \beta}+\sum_{i, j=1,2} \frac{m_{i j}}{v_{\varphi^{\prime}}} c_{X}\left(V_{N}\right)_{\alpha i}\left(V_{N}^{T}\right)_{j \beta}$,

where $M_{i}=y_{\varphi_{i}} v_{\varphi} / \sqrt{2}, \quad m_{i j} \equiv y_{\varphi_{i j}^{\prime}} v_{\varphi^{\prime}} / \sqrt{2}, \quad Q_{H}^{X} \equiv-4+$ $9\left|V_{N_{13}}\right|^{2}$ is the DM charge of hidden symmetry, and $Q_{H}^{\nu}=$ 1 is the active neutrino charge of hidden gauge symmetry. Notice here that we have used the unitarity of $V_{N}$ to derive $Q_{H}^{X} ; \sum_{a=1}^{3} V_{N_{1 a}}^{*} V_{N_{a 1}}^{T}=1$. The first and second terms induce the mode of the active neutrino final state via $Z^{\prime}$ vector boson exchange in the $s$ channel; the last two terms also provide a final state containing new scalar bosons from the second Higgs doublet. On the other hand, the third term induces the annihilation process in which the final state is GB via the diagrams with neutral fermions in the $t$ and $u$ channels. The relic density of DM is then given by $[18,19]$

$$
\Omega h^{2} \approx \frac{1.07 \times 10^{9}}{\sqrt{g_{*}\left(x_{f}\right)} M_{\mathrm{Pl}} J\left(x_{f}\right)[\mathrm{GeV}]},
$$

where $g^{*}\left(x_{f} \approx 25\right)$ is the degrees of freedom for relativistic particles at temperature $T_{f}=M_{X} / x_{f}$, $M_{\mathrm{Pl}} \approx 1.22 \times 10^{19} \mathrm{GeV}$, and $J\left(x_{f}\right)\left(\equiv \int_{x_{f}}^{\infty} d x \frac{\left\langle\sigma v_{\text {rel }}\right\rangle}{x^{2}}\right)$ is given by $[20,21]$

$$
\begin{gathered}
J\left(x_{f}\right)=\int_{x_{f}}^{\infty} d x\left[\frac{\int_{4 M_{X}^{2}}^{\infty} d s \sqrt{s-4 M_{X}^{2}}\left[W_{Z^{\prime}}(s)+W_{\alpha_{G}}(s)\right] K_{1}\left(\frac{\sqrt{s}}{M_{X}} x\right)}{16 M_{X}^{5} x\left[K_{2}(x)\right]^{2}}\right], \\
W_{Z^{\prime}}(s) \approx \frac{\left(s-4 M_{X}^{2}\right)}{8 \pi}\left|\frac{g_{H}^{2} Q_{H}^{X}}{s-m_{Z^{\prime}}^{2}+i m_{Z^{\prime}} \Gamma_{Z^{\prime}}}\right|^{2}\left(s+\frac{1}{2}\left(s-4 m_{\Phi}^{2}\right)\right), \\
W_{\alpha_{G}}(s) \simeq \frac{\left|\tilde{M}_{11}\right|^{4}}{64 \pi \tilde{v}_{\varphi \varphi^{\prime}}^{4}}\left[\left(3 s^{2}-4 M_{X}^{4}\right)\left(\frac{\pi}{2 s M_{X}^{2}} \sqrt{\frac{M_{X}^{4}}{4 s M_{X}^{2}-s^{2}}}-\frac{\tan ^{-1}\left[\frac{s-2 M_{X}^{2}}{\sqrt{s\left(4 M_{X}^{2}-s\right)}}\right]}{s^{3 / 2} \sqrt{4 M_{X}^{2}-s}}\right)-4\right],
\end{gathered}
$$

where we implicitly impose the kinematical constraint above, we take degenerate $H^{ \pm}\left(\phi_{R, I}\right)$ mass as $m_{\Phi}$, and the $X X \rightarrow Z^{\prime} Z^{\prime}$ process is omitted here for simplicity. Here, $Z^{\prime}$ can decay into $\nu_{R} \bar{\nu}_{R}, \psi_{\alpha} \psi_{\alpha}$, and $H^{+} H^{-}\left(\phi_{R} \phi_{I}\right)$ if kinematically allowed. The decay width of $Z^{\prime}$, which consists of $\Gamma_{Z^{\prime}} \equiv \Gamma_{Z^{\prime} \rightarrow \nu_{R} \bar{\nu}_{R}}+\Gamma_{Z^{\prime} \rightarrow X \bar{X}}+\Gamma_{Z^{\prime} \rightarrow H^{+} H^{-}}+\Gamma_{Z^{\prime} \rightarrow \phi_{R} \phi_{I}}$, is given by

$$
\begin{gathered}
\Gamma_{Z^{\prime} \rightarrow \nu_{R} \bar{\nu}_{R}}=\frac{g_{H}^{2} m_{Z^{\prime}}}{8 \pi}, \\
\Gamma_{Z^{\prime} \rightarrow X \bar{X}}=\frac{g_{H}^{2} m_{Z^{\prime}}}{96 \pi}\left|Q_{H}^{X}\right|^{2}\left(1-\frac{4 M_{X}^{2}}{m_{Z^{\prime}}^{2}}\right)^{3 / 2}, \\
\Gamma_{Z^{\prime} \rightarrow H^{+} H^{-}\left(\phi_{R} \phi_{I}\right)}=\frac{g_{H}^{2}}{48 \pi} m_{Z^{\prime}}\left(1-\frac{4 m_{\Phi}^{2}}{m_{Z^{\prime}}^{2}}\right)^{\frac{3}{2}},
\end{gathered}
$$

where we assume masses of $\psi_{2,3}$ are heavier than $m_{Z^{\prime}} / 2$ and $N_{c}^{f}$ is a color factor. Remember here that the $Z^{\prime}$ mass is given by $m_{Z^{\prime}}=g_{H} \sqrt{v_{\varphi}^{2}+\left(8 v_{\varphi^{\prime}}\right)^{2}}$ in Eq. (12).

In Fig. 1, we show the relic density in terms of $M_{X}$, where we fix the following parameters ${ }^{4}$ :

\footnotetext{
${ }^{4}$ In principle, one has to derive this mixing and its masses by diagonalizing $M_{N}$ in the neutral fermions. But here we expect any values can be taken, since all the mass parameters except the DM mass and its mixing are free.
}

$$
\begin{array}{rlrl}
g_{H} & =0.05, \quad\left|V_{N_{13}}\right|=0.1, \quad m_{Z^{\prime}}=250 \mathrm{GeV} \\
\tilde{v}_{\varphi \varphi^{\prime}} & =100 \mathrm{GeV}, \quad\left|\tilde{M}_{11}\right|=20 \mathrm{GeV} \\
M_{\psi_{2}} & =500 \mathrm{GeV}, & M_{\psi_{3}}=1000 \mathrm{GeV} \\
m_{\Phi} & =500 \mathrm{GeV} . & &
\end{array}
$$

The figure suggests the allowed range for $0.05 \leq g_{H}$,

$$
40 \mathrm{GeV} \lesssim M_{X} \lesssim 110 \mathrm{GeV} \text {, and } 125 \mathrm{GeV} \lesssim M_{X} \text {, }
$$

while for $g_{H} \leq 0.05$,

$$
M_{X} \lesssim 40 \mathrm{GeV}, \text { and } 110 \mathrm{GeV} \lesssim M_{X} \lesssim 125 \mathrm{GeV} \text {, }
$$

when all the parameters except $g_{H}$ are fixed and this region indicates that the observed relic density is obtained around resonant point $M_{X} \sim m_{Z^{\prime}} / 2$ where $m_{Z^{\prime}}$ is proportional to $g_{H}$. Here, we search for a parameter region satisfying the observed relic density in general, and we apply micrOMEGAs4.3.5 [22] to estimate the annihilation cross sections. Note that the $X X \rightarrow Z^{\prime} Z^{\prime}$ process is also included in the following analysis. Then, we scan the parameter region as follows: 


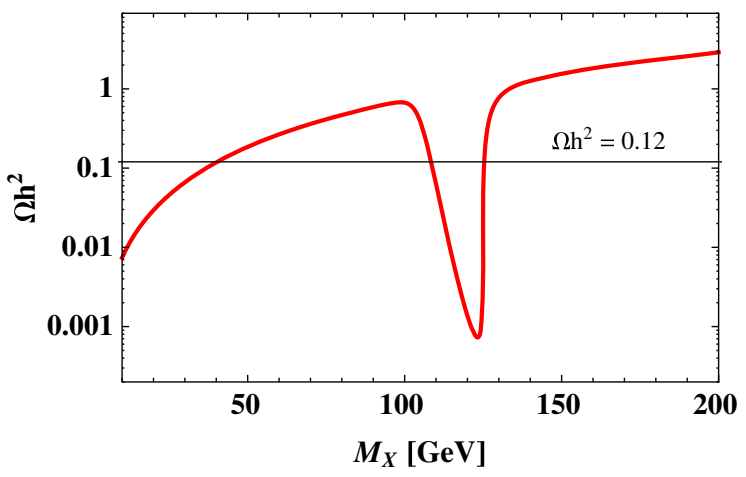

FIG. 1. The correlation between $M_{X}$ and $\Omega h^{2}$, where the horizontal black line is 0.12 . The other parameters are fixed as given in Eq. (23).

$M_{X} \in[10,1000] \mathrm{GeV}, \quad m_{Z^{\prime}} \in[100,2000] \mathrm{GeV}$,

$\frac{\tilde{M}_{\alpha \beta}}{\tilde{v}_{\varphi \varphi^{\prime}}} \in[0.025,0.4], \quad g_{H} \in[0.05,0.4]$,

$V_{13} \in[0.1,1 / \sqrt{2}], \quad M_{\psi_{1,2}} \in\left[M_{X}, 1500\right] \mathrm{GeV}$,

$m_{\Phi} \in[100,300] \mathrm{GeV}$.

In Fig. 2, we also show the parameter points on the $M_{X}-m_{Z^{\prime}}$ plane, which gives the relic density $0.11<$ $\Omega h^{2}<0.13$, fixing the other parameters as given in Eq. (26). We find that several specific regions can explain the relic density of DM:

(1) In the light $M_{X}$ region, the $X X \rightarrow \alpha_{G} \alpha_{G}$ process is the dominant one and insensitive to $m_{Z^{\prime}}$.

(2) The line-shaped region indicates $m_{Z^{\prime}} \sim 2 M_{X}$, in which the relic density is explained with a resonant effect.

(3) In the heavy $M_{X}$ region, the relic density can be explained by the $X X \rightarrow Z^{\prime} Z^{\prime}$ process with a relevant value of $g_{H}$.

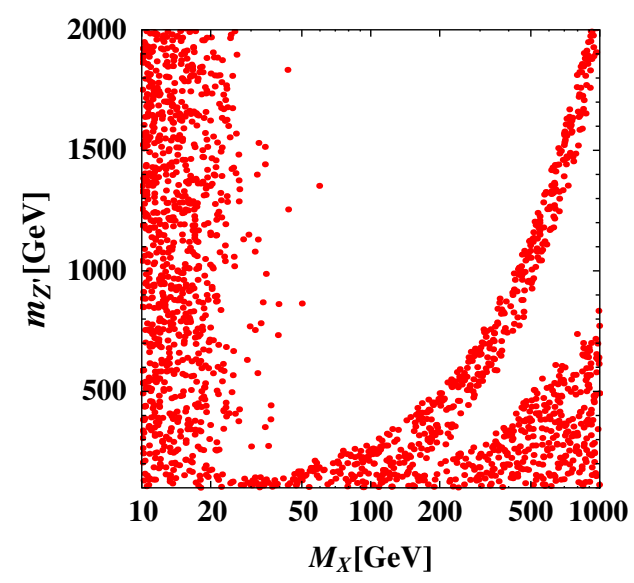

FIG. 2. The correlation between $M_{X}$ and $m_{Z^{\prime}}$ when the estimated relic density is $0.11<\Omega h^{2}<0.13$. The other parameters are fixed as given in Eq. (26).

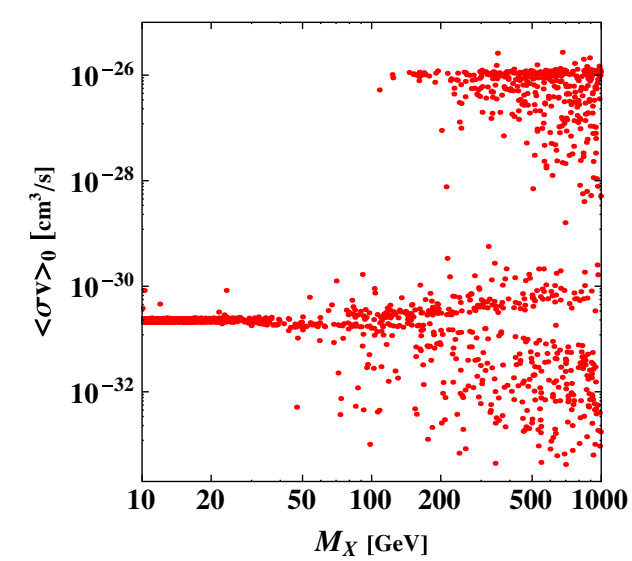

FIG. 3. The DM annihilation cross section at the current Universe for the parameter region that provides the correct relic density.

In addition, we show DM annihilation cross section at the current Universe for the parameter region giving the right relic density in Fig. 3. The cross section is suppressed for $\alpha_{G} \alpha_{G}$ and $Z^{\prime} Z^{\prime}$ modes, while it can be $\sim 10^{-26} \mathrm{~cm}^{3} / \mathrm{s}$ for $\nu_{R} \bar{\nu}_{R}$ and $H^{+} H^{-}\left(\phi_{R} \phi_{I}\right)$ modes. Since the ratio of the $H^{+} H^{-}$mode is around $10 \%$ in the latter case, our scenario is safe from constraints of indirect detection experiments, and it will be tested in future measurements of gamma-ray and neutrino flux from DM annihilation.

\section{CONCLUSION}

We have proposed a neutrinophilic two-Higgs-doublet model with hidden local $U(1)_{H}$ symmetry introducing right-handed neutrinos and exotic SM singlet fermions for anomaly cancellation. The active neutrino masses are Dirac type induced by the tiny VEV of a neutrinophilic Higgs doublet, the interaction with other SM fermions of which is forbidden by the $U(1)_{H}$ symmetry. We formulated the boson and fermion sectors where a fermionic DM candidate naturally arises as the lightest mass eigenstate of an exotic fermion since it is stable due to a remnant symmetry even after the spontaneous symmetry breaking. Then, the DM candidate interacts with active neutrinos by exchanging the $Z^{\prime}$ boson from $U(1)_{H}$. Moreover, a physical GB is induced as a consequence of two types of gauge singlet scalar fields and contributes to the DM annihilation processes determining the relic density. Then, we analyzed the relic density of DM, within the safe range of direct detection searches, and found another allowed range with lighter DM mass that directly comes from the contribution of the GB mode in addition to the resonant allowed range via the $Z^{\prime}$ boson.

\section{ACKNOWLEDGMENTS}

H. O. is sincerely grateful for the Korea Institute for Advanced Study (KIAS) members. 


\section{APPENDIX: DERIVING $\alpha_{G}$ AND $\alpha_{N G}$ IN EQS. (10) AND (11)}

Here, we derive the NG boson $\alpha_{N G}$ and physical Goldstone boson $\alpha_{G}$ from $\varphi$ and $\varphi^{\prime}$ expressed as in Eq. (4), in which mixing between $\phi_{I}$ is ignored, assuming tiny $v_{\phi}$. The covariant derivative of $\varphi\left(\varphi^{\prime}\right)$ is given by

$$
D_{\mu} \varphi\left(\varphi^{\prime}\right)=e^{i \frac{\alpha\left(\alpha^{\prime}\right)}{\varphi\left(\varphi^{\prime}\right)}}\left(\partial_{\mu}+i \frac{1}{v_{\varphi\left(\varphi^{\prime}\right)}} \partial_{\mu} \alpha\left(\alpha^{\prime}\right)-i g_{H} Q_{\varphi\left(\varphi^{\prime}\right)} Z_{\mu}^{\prime}\right) r_{\varphi}\left(r_{\varphi^{\prime}}\right)
$$

where $Q_{\varphi\left(\varphi^{\prime}\right)}=1(8)$ is the $U(1)_{H}$ charge of $\varphi\left(\varphi^{\prime}\right)$ and $r_{\varphi\left(\varphi^{\prime}\right)}=\left[v_{\varphi\left(\varphi^{\prime}\right)}+\varphi_{R}\left(\varphi_{R}^{\prime}\right)\right] / \sqrt{2}$. We then have

$$
\begin{aligned}
\mathcal{L}_{\text {kinetic }}= & \left(D_{\mu} \varphi\right)^{\dagger}\left(D^{\mu} \varphi\right)+\left(D_{\mu} \varphi^{\prime}\right)^{\dagger}\left(D^{\mu} \varphi^{\prime}\right) \\
= & \frac{1}{2} \partial_{\mu} \varphi_{R} \partial^{\mu} \varphi_{R}+\frac{1}{2} \partial_{\mu} \varphi_{R}^{\prime} \partial^{\mu} \varphi_{R}^{\prime}+\frac{1}{2}\left(v_{\varphi}^{2}+2 v_{\varphi} \varphi_{R}+\varphi_{R}^{2}\right)\left(\frac{1}{v_{\varphi}^{2}} \partial_{\mu} \alpha \partial^{\mu} \alpha-\frac{2 g_{H} Q_{\varphi}}{v_{\varphi}} \partial_{\mu} \alpha Z^{\prime \mu}+g_{H}^{2} Q_{\varphi}^{2} Z_{\mu}^{\prime} Z^{\prime \mu}\right) \\
& +\frac{1}{2}\left(v_{\varphi^{\prime}}^{2}+2 v_{\varphi^{\prime}} \varphi_{R}^{\prime}+\varphi_{R}^{\prime 2}\right)\left(\frac{1}{v_{\varphi^{\prime}}^{2}} \partial_{\mu} \alpha^{\prime} \partial^{\mu} \alpha^{\prime}-\frac{2 g_{H} Q_{\varphi^{\prime}}}{v_{\varphi^{\prime}}} \partial_{\mu} \alpha^{\prime} Z^{\prime \mu}+g_{H}^{2} Q_{\varphi^{\prime}}^{2} Z_{\mu}^{\prime} Z^{\prime \mu}\right) .
\end{aligned}
$$

Here, we add the gauge fixing term,

$$
\begin{aligned}
\mathcal{L}_{G} & =-\frac{1}{2} G^{2}, \\
G & =\frac{1}{\sqrt{\xi}}\left(\partial_{\mu} Z^{\prime \mu}+\xi g_{H} Q_{\varphi} v_{\varphi} \alpha+\xi g_{H} Q_{\varphi^{\prime}} v_{\varphi^{\prime}} \alpha^{\prime}\right),
\end{aligned}
$$

where $\xi$ is a gauge fixing parameter. Combining Eqs. (A2) and (A3), we obtain mass terms for $Z^{\prime}$ and $\alpha\left(\alpha^{\prime}\right)$ such that

$$
\mathcal{L}_{M}=\frac{1}{2} g_{H}^{2}\left(Q_{\varphi}^{2} v_{\varphi}^{2}+Q_{\varphi^{\prime}}^{2} v_{\varphi^{\prime}}^{2}\right) Z_{\mu}^{\prime} Z^{\prime \mu}-\frac{1}{2} \xi g_{H}^{2}\left(Q_{\varphi}^{2} v_{\varphi}^{2}+Q_{\varphi^{\prime}}^{2} v_{\varphi^{\prime}}^{2}\right)\left[\frac{Q_{\varphi} v_{\varphi}}{\sqrt{Q_{\varphi}^{2} v_{\varphi}^{2}+Q_{\varphi^{\prime}}^{2} v_{\varphi^{\prime}}^{2}}} \alpha+\frac{Q_{\varphi^{\prime}} v_{\varphi^{\prime}}}{\sqrt{Q_{\varphi}^{2} v_{\varphi}^{2}+Q_{\varphi^{\prime}}^{2} v_{\varphi^{\prime}}^{2}}} \alpha^{\prime}\right]^{2}
$$

Thus, the second term corresponds to the gauge-dependent mass term for the NG boson, and the physical Goldstone boson state is orthogonal to the NG boson one. Therefore, the $\alpha_{N G}$ and $\alpha_{G}$ are given as Eqs. (10) and (11).

[1] S. M. Davidson and H. E. Logan, Phys. Rev. D 80, 095008 (2009).

[2] F. Wang, W. Wang, and J. M. Yang, Europhys. Lett. 76, 388 (2006).

[3] S. Baek and T. Nomura, J. High Energy Phys. 03 (2017) 059.

[4] P. A. N. Machado, Y. F. Perez, O. Sumensari, Z. Tabrizi, and R. Z. Funchal, J. High Energy Phys. 12 (2015) 1.

[5] J. C. Montero and V. Pleitez, Phys. Lett. B 675, 64 (2009).

[6] E. Ma and R. Srivastava, Phys. Lett. B 741, 217 (2015).

[7] S. Singirala, R. Mohanta, and S. Patra, arXiv:1704.01107.

[8] T. Nomura and H. Okada, arXiv:1705.08309.

[9] T. Nomura and H. Okada, Eur. Phys. J. C 78, 189 (2018).

[10] V. De Romeri, E. Fernandez-Martinez, J. Gehrlein, P. A. N. Machado, and V. Niro, J. High Energy Phys. 10 (2017) 169.

[11] E. Bertuzzo, P. A. N. Machado, Z. Tabrizi, and R. Zukanovich Funchal, J. High Energy Phys. 11 (2017) 004.

[12] M. D. Campos, D. Cogollo, M. Lindner, T. Melo, F. S. Queiroz, and W. Rodejohann, J. High Energy Phys. 08 (2017) 092.
[13] D. V. Forero, M. Tortola, and J. W. F. Valle, Phys. Rev. D 86, 073012 (2012).

[14] D. S. Akerib et al. (LUX Collaboration), Phys. Rev. Lett. 118, 021303 (2017).

[15] E. Aprile et al. (XENON Collaboration), Phys. Rev. Lett. 119, 181301 (2017).

[16] X. Cui et al. (PandaX-II Collaboration), Phys. Rev. Lett. 119, 181302 (2017).

[17] P. A. R. Ade et al. (Planck Collaboration), Astron. Astrophys. 571, A16 (2014).

[18] K. Griest and D. Seckel, Phys. Rev. D 43, 3191 (1991).

[19] J. Edsjo and P. Gondolo, Phys. Rev. D 56, 1879 (1997).

[20] K. Nishiwaki, H. Okada, and Y. Orikasa, Phys. Rev. D 92 , 093013 (2015).

[21] P. Ko, T. Nomura, H. Okada, and Y. Orikasa, Phys. Rev. D 94, 013009 (2016).

[22] G. Belanger, F. Boudjema, A. Pukhov, and A. Semenov, Comput. Phys. Commun. 192, 322 (2015). 